Dendrobaena und Eisenia. Ihre Arten leben vorwiegend im oberen Waldboden und verzehren leicht verrottete Laub- und Nadelstren. Zur zweiten Gruppe gehören die Gattungen Allolobophora und Octolasium. Thre Vertreter sind schwärzlich, grau, bläulich oder grünlich pigmentiert. Sie benötigen ein viel weitgehender zersegtes Pflanzenmaterial als die roten Arten und leben vornehmlich in tieferen Bodenschichten reiner Laub- oder Laubmischwälder. Kurzdiagnosen der zehn waldbewohnenden Regenwurmarten werden gegeben und es wird auch über Körperbau, Fortpflanzung und Lebensdauer berichtet. Die einheimischen Arten bringen „unter normalen Boden- und Klimaverhältnissen" jährlich eine Generation hervor. Im Freiland werden die Tiere kaum über 2 Jahre alt. Abschließend wird ,,die Bedeutung der Regenwürmer für den Lebenshaushalt des Waldes" gewürdigt. Ihr Nutzen liegt in der mechanischen - (Bodendurchlüftung, Bodendurchmischung) und chemischen Bodenverbesserung. Unter guten Bodenverhältnissen werden pro Jahr und Hektar rund 800 Doppelzentner Wurmkot oberflächlich abgesetzt, "Dies entspricht gewichtsmäßig einer starken Stallmistdüngung, zweimal jährlich gegeben." $\quad$ O. Eichhorn

\section{BIOLOGISCHE REGELUNG}

Zomorrodi, M. A., 1959: La Lutte Biologique Contre La Punaise Du Blé Eurygaster Integriceps Put. Par Microphanurus Semistriatus Nees. En Iran. Rev. Path. Végétale Ent. Agric. France XXXVIII, 3, 167-174.

Vor 12 Jahren wurde im Iran mit der biologischen Bekämpfung der Getreidewanze Eurygaster integriceps begonnen, und zwar mit Hilfe des hier weit verbreiteten Eiparasiten Microphanurus semistriatus (Encyrtidae). Der Autor studierte die Biologie des Parasiten und ermittelte die besten Bedingungen für dessen Zucht. Weiterhin wurden diejenigen Faktoren ermittelt, unter denen man am besten die Eier von Eurygaster, die von M. semistriatus parasitiert werden sollen, gewinnen kann. Für die Eiablage des Schädlings sind eine Temperatur von $26-30^{\circ} \mathrm{C}$ und eine rel. Luftfeuchtigkeit von $60-70 \%$ optimal. Das Eurygasterweibchen legt seine Eier in den Zuchten an schmale Papierstreifen ab; die Gelege werden später auf Papierbogen gesammelt. Fünf solcher Bogen werden mit einigen Microphanurusweibchen (1 Weibchen auf 30 Eurygaster-Eier) in Gefäße von $40 \times 20 \times 20 \mathrm{~cm}$ gebracht, in denen die nötige Luftfeuchtigkeit durch Einlegen von mit Wasser getränlkten Baumwollpfropfen erzielt wird. 207 Millionen Microphanurus-Exemplare wurden 1955 auf diese Weise in Ispahan gezüchtet. Mit Hilfe der so gewonnenen Parasiten konnte der Eurygasterbefall auf den behandelten Getreideffächen stark reduziert werden.

V. Schefer-Immel

Wilbert, H., 1959: Apanteles glomeratus (L.) als Parasit von Aporia crataegi (L.) (Hymenoptera: Braconidae). Beitr. Ent. 9, 7/8, 874-898.

Es wird über einige biologische Daten für Apanteles glomeratus an Aporia crataegi berichtet, so iber den Zeitpunkt der Eiablage, die für die Eiablage in Frage kommenden Stadien des Wirtes und den Zeitpunkt des Schlüpfens der Imagines des Parasiten. Letzterer liegt in der zweiten Maihälfte, in einer Zeit also, zu der noch keine belegungsfähigen Baumweißlingsraupen vorhanden sind. Die Zwischenzeit wird anscheinend durch eine weitere Generation an Kohlweißling überbrückt. A, glomeratus kann seinen Jahreszylilus nicht allein an Aporia crataegi vollenden, da die Embryonalentwicklung dieses Wirtes zu lange dauert und außerdem das Wachstum der Angehörigen einer Baumweißlingspopulation zu einheitlich ist. A. glomeratus kann, da er hier abwechselnd zwei Wirte befällt, sich bei einer Massenvermehrung des einen kaum dessen Gradation durch entsprechende eigene Zunahme anpassen und damit auch keine entscheidende Beeinflussung auf eine Massenvermehrung von $A$. crataegi haben. Der Parasit könnte höchstens dazu beitragen, die Population des Schädlings in normalen Jahren niedrig zu halten.

V. Schefer-Immel

Coutin, R. und A. Colombin, 1960: Les Principaux Parasites De Laspeyresia Pomonella L. Dans Le Bassin Parisien. Rev. Path. Vegetale Ent. Agric. France XXXIX, I, $35-45$.
Es wurden 14 Parasitenarten aus dem Apfelwickler, Laspeyresia pomonella, erzogen, von denen folgende 4. Arten im Nordwesten Frankreichs häufig waren: die Ichneumoniden Pristomerus vulnerator Panz. und Trichomma enecator Rossi, sowie die Braconide Ascogaster quadridentatus W/sm. und die Tachine Elodia tragica Fall. Jede Art wird durch das bestimmte Verhältnis der Zeit ihrer Entwicklung in Beziehung zu derjenigen ihres Wirtes charakterisiert. Die Befallsstärke war nirgends besonders hoch, am niedrigsten aber in Obstanlagen in der Nähe von Städten oder in solchen, die einer sorgfältigen chemischen Behandlung unterzogen worden waren.

V. Schefer-Immel

\section{Kleine Mitteilungen}

Spanischer Verdienstorden für Dr. Drees

Für Verdienste um die internationale Zusammenarbeit auf dem Gebiet des Pflanzenschutzes ist Ministerialrat Dr. Drees, Bundesministerium für Ernährung, Landwirtschaft und Forsten, Bonn, von der spanischen Regierung der "Orden Civil del Merito Agricola" (Halsorden) verliehen worden.

\section{Dr.-Fritz-Merck-Preis}

Anläßlich des 70. Geburtstages von Herrn Dr. Fritz Merck, Ehrensenator der Justus-Liebig-Universität in GieBen, hat der Vorstand der E. Merck AG, Darmstadt, den "Dr.-Fritz-Merck-Preis" ins Leben gerufen.

Der Preis wird für besondere wissenschaftliche Leistungen verliehen, welche die Aufklärung oder die Beseitigung von Störungen in der Biosphäre zum Gegenstand haben. Hierunter fallen Arbeiten aus den einschlägigen naturwissenschaftlichen Disziplinen, insbesondere aus dem Gebiet des Pflanzenschutzes.

Zweck der Stiftung ist es, die Arbeit aufstrebender, vor allem jüngerer Fachwissenschaftler deutscher Staatsangehörigkeit oder mit Wohnsitz in Deutschland anzuerkennen und zu fördern. Angestellten von Industrie- und Handelsfirmen wird der Preis nicht verliehen.

Die Stiftung ist mit jährlich DM $10000,-$ ausgestattet. Uther die Zuerkennung des Preises, der im ganzen oder geteilt vergeben werden kann, entscheidet das Kuratorium. Preisträger können Einzelpersonen wie auch Arbeitsgemeinschaften sein.

Bewerber werden entweder von den Mitgliedern des Kuratoriums oder durch Empfehlung eines anerkannten Fachvertreters benannt. Sie können auch selbst Antrag auf Zuerkennung des Preises stellen. Im letzteren Falle muls die wissenschaftliche Arbeit unter Beifügung des Gutachtens eines anerkannten Fachvertreters vorgelegt werden.

Bewerbungen sind ausschlieBlich an das Sekretariat des Dr.-Fritz-Merck-Preises, z. H. von Dr. H. Laber, Darmstadt, Frankfurter Straße 250, Fernruf 8021, Apparat 212 , zu richten.

Anträge, die bis zum 1. Mai eines jeden Kalenderjahres dem Kuratorium im ordentilichen Geschäftsweg zugehen, werden bis spätestens 1 . November des gleichen Jahres geprüft und endgültig entschieden.

Die Preisträger werden alljährlich anläßlich der Feier der Justus-Liebig-Universität Gießen öffentlich bekanntgegeben.

Die Deutsche Landwirtschafts-Gesellschaft

- Feierstunde am 10. Dezember 1960 in der Paulskirche Frankfurt am Main -

Am 11. Dezember 1885 wurde die Deutsche Landwirtschafts-Gesellschaft (DLG) im Englischen Haus in der Mohrenstraße zu Berlin von dem Dichter-Ingenieur Max Eyth gegrïndet. Die DLG hat in Anwesenheit von Bundespräsident Dr. h. c. Heinrich L übke am 10. Dezember 1960 ihrer Gründung vor $75 \mathrm{Jahren}$ in einer Feierstunde in der Paulskirche zu Frankfurt am Main gedacht. Die Spitzen der öffentlichkeit und der deutschen Landwirtschaft, Freunde, Förderer und Mitarbeiter der Deutschen Landwirtschafts-Gesellschaft haben an dieser Feiexstunde teilgenommen.

Holzmesse 1961 - Gesamtschau der Forst- und Holzwirtschaft - findet vom 20. bis 29. Oktober 1961 in Düsseldorf statt. 